\title{
An Oscillation Test for Solutions of Second-Order Neutral Differential Equations of Mixed Type
}

\author{
Osama Moaaz ${ }^{1, *(\mathbb{D})}$, Ali Muhib ${ }^{1,2}$ and Shyam S. Santra ${ }^{3}(\mathbb{D}$ \\ 1 Department of Mathematics, Faculty of Science, Mansoura University, Mansoura 35516, Egypt \\ 2 Department of Mathematics, Faculty of Education-Al-Nadirah, Ibb University, Ibb 70270, Yemen; \\ muhib39@yahoo.com \\ 3 Department of Mathematics, JIS College of Engineering, Kalyani 741235, India; shyam01.math@gmail.com \\ * Correspondence: o_moaaz@mans.edu.eg
}

check for updates

Citation: Moaaz, O.; Muhib; A.; Santra S.S. An Oscillation Test for Solutions of Second-Order Neutral Differential Equations of Mixed Type. Mathematics 2021, 9, 1634.

https://doi.org/10.3390/math9141634

Academic Editors: Eva Kaslik and Christopher Goodrich

Received: 20 January 2021 Accepted: 19 February 2021 Published: 11 July 2021

Publisher's Note: MDPI stays neutral with regard to jurisdictional claims in published maps and institutional affiliations.

\begin{abstract}
It is easy to notice the great recent development in the oscillation theory of neutral differential equations. The primary aim of this work is to extend this development to neutral differential equations of mixed type (including both delay and advanced terms). In this work, we consider the second-order non-canonical neutral differential equations of mixed type and establish a new single-condition criterion for the oscillation of all solutions. By using a different approach and many techniques, we obtain improved oscillation criteria that are easy to apply on different models of equations.
\end{abstract}

Keywords: non-canonical differential equations; second-order; neutral delay; mixed type; oscillation criteria

\section{Introduction}

This paper discusses the oscillatory behavior of solutions of second-order neutral differential equations of mixed type:

$$
\begin{aligned}
\left(r(s)\left(\left(x(s)+p_{1}(s) x\left(\varrho_{1}(s)\right)+p_{2}(s) x\left(\varrho_{2}(s)\right)\right)^{\prime}\right)^{\alpha}\right)^{\prime} & \\
+ & q_{1}(s) x^{\alpha}\left(\theta_{1}(s)\right)+q_{2}(s) x^{\alpha}\left(\theta_{2}(s)\right)=0
\end{aligned}
$$

where $s \geq s_{0}$. Throughout this paper, we assume the following:

(C1) $\alpha \in Q_{o d d}^{+}:=\left\{a / b: a, b \in \mathbb{Z}^{+}\right.$are odd $\}$;

(C2) $r \in C\left(\left[s_{0}, \infty\right),(0, \infty)\right) r^{\prime}(s)>0$, and $\int_{s_{0}}^{\infty} r^{-1 / \alpha}(\xi) \mathrm{d} \xi<\infty$, where $C(I, J)$ is the set of all continuous real-valued functions $F: I \rightarrow J$;

(C3) $\varrho_{1}, \varrho_{2}, \theta_{1}, \theta_{2} \in C\left(\left[s_{0}, \infty\right), \mathbb{R}\right), \varrho_{1}(s) \leq s, \varrho_{2}(s) \geq s, \theta_{1}(s) \leq s, \theta_{2}(s) \geq s$, and $\varrho_{1}(s), \varrho_{2}(s), \theta_{1}(s), \theta_{2}(s) \rightarrow \infty$ as $s \rightarrow \infty$;

(C4) $p_{1}, p_{2}, q_{1}, q_{2} \in C\left(\left[s_{0}, \infty\right),[0, \infty)\right)$ and $q_{1}, q_{2}$ are not identically zero for large $s$.

Let $x$ be a real-valued function defined for all $s$ in a real interval $\left[s_{x}, \infty\right), s_{x} \geq s_{0}$, which has the properties

$$
x+p_{1} \cdot x \circ \varrho_{1}+p_{2} \cdot x \circ \varrho_{2} \in C^{1}\left(\left[s_{x}, \infty\right), \mathbb{R}\right)
$$

and

$$
r \cdot\left(x+p_{1} \cdot x \circ \varrho_{1}+p_{2} \cdot x \circ \varrho_{2}\right)^{\prime} \in C^{1}\left(\left[s_{x}, \infty\right), \mathbb{R}\right) .
$$

Then, $x$ is called a solution of (1) on $\left[s_{x}, \infty\right)$ if $x$ satisfies (1) for all $s \geq s_{x}$. We will consider only the solutions of (1) that exist on some half-line $\left[s_{x}, \infty\right)$ for $s_{x} \geq s_{0}$ and satisfy the condition

$$
\sup \left\{|x(s)|: s_{c} \leq s<\infty\right\}>0 \text { for any } s_{c} \geq s_{x} .
$$

A nontrivial solution $x$ of any differential equation is said to be oscillatory if it has arbitrary large zeros; otherwise, it is said to be non-oscillatory. 
The oscillation and asymptotic behavior of solutions to various classes of delay and advanced differential equations have been widely discussed in the literature. For second-order delay equations, the studies found in [1-5] were concerned with studying the oscillatory behavior of the equation:

$$
\left(r(s)\left(\left(x(s)+p_{1}(s) x\left(\varrho_{1}(s)\right)\right)^{\prime}\right)^{\alpha}\right)^{\prime}+q_{1}(s) x^{\alpha}\left(\theta_{1}(s)\right)=0,
$$

with the canonical operator $\pi\left(s_{0}\right)=\infty$, where

$$
\pi(s):=\int_{s_{0}}^{s} r^{-1 / \alpha}(\xi) \mathrm{d} \xi
$$

One can find developments and comparisons of the oscillation criteria of (2) in the recently published paper by Moaaz et al. [4] for a non-canonical case, that is,

$$
\int_{s_{0}}^{\infty} r^{-1 / \alpha}(\xi) \mathrm{d} \xi<\infty .
$$

Bohner et al. [6] simplified and improved the previous results found by Agarwal et al. [7] and Han et al. [8]. For more general equations and more accurate results, see [9,10].

For second-order advanced equations, Chatzarakis et al. [11,12] studied the asymptotic behavior of the equation:

$$
\left(r(s)\left(x(s)^{\prime}\right)^{\alpha}\right)^{\prime}+q_{2}(s) x^{\alpha}\left(\theta_{2}(s)\right)=0
$$

in the non-canonical case, and improved a number of pre-existing results.

Although there are many results of studies of the oscillation of solutions of delay differential equations, the results that concern the study of mixed equations are few-see, for example [13-24]. By using the Riccati transformation technique, Arul and Shobha [13] obtained some sufficient conditions for oscillation of the equation:

$$
\left(r(s)(x(s)+a(s) x(s-\varrho)+b(s) x(s+\delta))^{\prime}\right)^{\prime}+q(s) f(x(\theta(s)))=0,
$$

where $0 \leq a(s) \leq a<\infty, 0 \leq b(s) \leq b<\infty$, and $f(u) / u \geq k>0$. Dzurina et al. [22] established some criteria for the oscillation of the equation

$$
\left(x(s)+p_{1} x\left(s-\varrho_{1}\right)+p_{2} x\left(s+\varrho_{2}\right)\right)^{\prime \prime}=q_{1}(s) x\left(s-\theta_{1}\right)+q_{2}(s) x\left(s+\theta_{2}\right),
$$

where $\varrho_{i}, \theta_{i} \geq 0$ are constants, $q_{i}$ is nonnegative, and $i=1,2$. Tunc et al. [24] studied the oscillatory behavior of solutions of the equation:

$$
\left(r(s)\left(\left(x(s)+p_{1}(s) x\left(\varrho_{1}(s)\right)+p_{2}(s) x\left(\varrho_{2}(s)\right)\right)^{\prime}\right)^{\alpha}\right)^{\prime}+q(s) x^{\alpha}(\theta(s))=0,
$$

in the canonical case $\pi\left(s_{0}\right)=\infty$, and considered the cases:

$$
\text { (i) } p_{1}(s) \geq 0, p_{2}(s) \geq 1 \text { and } p_{2}(s) \neq 1 \text { eventually }
$$

and

(ii) $p_{2}(s) \geq 0, p_{1}(s) \geq 1$ and $p_{2}(s) \neq 1$ eventually.

Thandapani et al. [23] considered the equation

$$
\left(\left(x(s)+p_{1} x\left(s-\varrho_{1}\right)+p_{2} x\left(s+\varrho_{2}\right)\right)^{\alpha}\right)^{\prime \prime}+q_{1}(s) x^{\beta}\left(s-\theta_{1}\right)+q_{2}(s) x^{\gamma}\left(s+\theta_{2}\right)=0,
$$

where $\alpha, \beta$, and $\gamma$ are the ratios of odd positive integers, and established some sufficient conditions for the oscillation of all of the solutions. For more results, techniques, and 
approaches that deal with the oscillation of delay differential equations of higher orders, see [25-33].

The objective of this paper is to study the oscillatory and asymptotic properties of a class of delay differential equations of mixed neutral type with the non-canonical operator. The oscillation criteria are obtained via only one condition, and hence, they are easy to apply. Moreover, by using generalized Riccati substitution, we get new criteria that improve some of the results reported in the literature. An example is provided to illustrate the significance of the main results.

\section{Preliminary Results}

In the following, we present the notations used in this study:

- $\quad$ For the continuous function $r$, we define the integral operator $\kappa(u, v)$ for $u<v$ as

$$
\kappa(u, v):=\int_{u}^{v} r^{-1 / \alpha}(\delta) \mathrm{d} \delta
$$

- $\quad$ For any solution $x$ of (1), we define the corresponding function $v$ as

$$
v(s):=x(s)+p_{1}(s) x\left(\varrho_{1}(s)\right)+p_{2}(s) x\left(\varrho_{2}(s)\right), \text { for } s \geq s_{0} .
$$

- $\quad$ Briefly, we use the notations

$$
\begin{aligned}
B_{1}(s): & =1-p_{1}(s) \frac{\kappa\left(\varrho_{1}(s), \infty\right)}{\kappa(s, \infty)}-p_{2}(s) \\
H(s) & :=q_{1}(s) B_{2}^{\alpha}\left(\theta_{1}(s)\right)+q_{2}(s) B_{2}^{\alpha}\left(\theta_{2}(s)\right) \\
G(s): & =q_{1}(s) B_{1}^{\alpha}\left(\theta_{1}(s)\right)+q_{2}(s) B_{1}^{\alpha}\left(\theta_{2}(s)\right)
\end{aligned}
$$

and

$$
B_{2}(s):=1-p_{1}(s)-p_{2}(s) \frac{\kappa\left(s_{1}, \varrho_{2}(s)\right)}{\kappa\left(s_{1}, s\right)}, \text { for } s \geq s_{1} \geq s_{0}
$$

Lemma 1 ([6], Lemma 2.6). Assume that $\Theta(v):=A v-B(v-C)^{(\alpha+1) / \alpha}$, where $A, B$, and $C$ are real constants, $B>0$, and $\alpha \in Q_{\text {odd }}^{+}$. Then, the maximum value of $\Theta$ on $\mathbb{R}$ at $v^{*}=$ $C+(\alpha A /((\alpha+1) B))^{\alpha}$ is

$$
\Theta\left(v^{*}\right) \leq \max _{v \in \mathbb{R}} \Theta(v)=A C+\frac{\alpha^{\alpha}}{(\alpha+1)^{\alpha+1}} A^{\alpha+1} B^{-\alpha} .
$$

Lemma 2. Let $x$ be a positive solution of (1). If $v$ is decreasing, then

$$
\left(\frac{v(s)}{\kappa(s, \infty)}\right)^{\prime} \geq 0
$$

eventually. Further, if $v$ is increasing, then

$$
\left(\frac{v(s)}{\kappa\left(s_{1}, s\right)}\right)^{\prime} \leq 0
$$

for all $s \geq s_{1} \geq s_{0}$.

Proof. Suppose that (1) has a positive solution $x$ on $\left[s_{0}, \infty\right)$. Obviously, $v(s) \geq x(s)>0$ for all $s \geq s_{1} \geq s_{0}$. Thus, from (1), we get

$$
\left(r(s)\left(v^{\prime}(s)\right)^{\alpha}\right)^{\prime}=-q_{1}(s) x^{\alpha}\left(\theta_{1}(s)\right)-q_{2}(s) x^{\alpha}\left(\theta_{2}(s)\right) \leq 0 .
$$


Hence, $r(s)\left(v^{\prime}(s)\right)^{\alpha}$ is non-increasing, and so $v^{\prime}(s)$ has a constant sign for $s \geq s_{1}$. Assume that $v^{\prime}(s)<0$ on $\left[s_{1}, \infty\right)$. Then,

$$
v(s) \geq-\int_{s}^{\infty} r^{-1 / \alpha}(\xi) r^{1 / \alpha}(\xi) v^{\prime}(\xi) \mathrm{d} \xi \geq-\kappa(s, \infty) r^{1 / \alpha}(s) v^{\prime}(s)
$$

and so,

$$
\left(\frac{v(s)}{\kappa(s, \infty)}\right)^{\prime}=\frac{\kappa(s, \infty) v^{\prime}(s)+r^{-1 / \alpha}(s) v(s)}{(\kappa(s, \infty))^{2}} \geq 0 .
$$

Next, assume that $v^{\prime}(s)>0$ on $\left[s_{1}, \infty\right)$. Hence, we obtain

$$
v(s) \geq \int_{s_{1}}^{s} r^{-1 / \alpha}(\xi) r^{1 / \alpha}(\xi) v^{\prime}(\xi) \mathrm{d} \xi \geq \kappa\left(s_{1}, s\right) r^{1 / \alpha}(s) v^{\prime}(s),
$$

and it follows that

$$
\left(\frac{v(s)}{\kappa\left(s_{1}, s\right)}\right)^{\prime}=\frac{\kappa\left(s_{1}, s\right) v^{\prime}(s)-r^{-1 / \alpha}(s) v(s)}{\kappa^{2}\left(s_{1}, s\right)} \leq 0 .
$$

Thus, the proof is complete.

\section{Main Results}

Theorem 1. Assume that $H(s) \geq G(s)>0$. If

$$
\limsup _{s \rightarrow \infty} \int_{s_{1}}^{s} \frac{1}{r^{1 / \alpha}(u)}\left(\int_{s_{1}}^{u} G(\xi) \kappa^{\alpha}\left(\theta_{2}(\xi), \infty\right) \mathrm{d} \xi\right)^{1 / \alpha} \mathrm{d} u=\infty,
$$

for $s_{1} \geq s_{0}$, then all solutions of (1) are oscillatory.

Proof. Assume the contrary: that (1) has a non-oscillatory solution $x$ on $\left[s_{0}, \infty\right)$. Without loss of generality (since the substitution $y=-x$ transforms (1) into an equation of the same form), we suppose that $x$ is an eventually positive solution. Then, there exists $s_{1} \geq s_{0}$ such that $x\left(\varrho_{1}(s)\right), x\left(\varrho_{2}(s)\right), x\left(\theta_{1}(s)\right)$, and $x\left(\theta_{2}(s)\right)$ are positive for all $s \geq s_{1}$. Thus, from (1) and the definition of $v$, we note that $v(s) \geq x(s)>0$ and $r(s)\left(v^{\prime}(s)\right)^{\alpha}$ is non-increasing. Hence, $v^{\prime}>0$ or $v^{\prime}<0$ eventually.

Assume that $v^{\prime}(s)<0$ on $\left[s_{1}, \infty\right)$. By using Lemma 2, we have

$$
v\left(\varrho_{1}(s)\right) \leq \frac{\kappa\left(\varrho_{1}(s), \infty\right)}{\kappa(s, \infty)} v(s)
$$

based on the fact that $\varrho_{1}(s) \leq s$. Therefore,

$$
\begin{aligned}
x(s) & =v(s)-p_{1}(s) x\left(\varrho_{1}(s)\right)-p_{2}(s) x\left(\varrho_{2}(s)\right) \\
& \geq v(s)-p_{1}(s) v\left(\varrho_{1}(s)\right)-p_{2}(s) v\left(\varrho_{2}(s)\right) \\
& \geq\left(1-p_{1}(s) \frac{\kappa\left(\varrho_{1}(s), \infty\right)}{\kappa(s, \infty)}-p_{2}(s)\right) v(s) \\
& =B_{1}(s) v(s) .
\end{aligned}
$$

Hence, (1) becomes

$$
\left(r(s)\left(v^{\prime}(s)\right)^{\alpha}\right)^{\prime} \leq-q_{1}(s) B_{1}^{\alpha}\left(\theta_{1}(s)\right) v^{\alpha}\left(\theta_{1}(s)\right)-q_{2}(s) B_{1}^{\alpha}\left(\theta_{2}(s)\right) v^{\alpha}\left(\theta_{2}(s)\right),
$$


and since $\theta_{1}(s) \leq \theta_{2}(s)$, we have

$$
\begin{aligned}
\left(r(s)\left(v^{\prime}(s)\right)^{\alpha}\right)^{\prime} & \leq-q_{1}(s) B_{1}^{\alpha}\left(\theta_{1}(s)\right) v^{\alpha}\left(\theta_{2}(s)\right)-q_{2}(s) B_{1}^{\alpha}\left(\theta_{2}(s)\right) v^{\alpha}\left(\theta_{2}(s)\right) \\
& \leq-\left(q_{1}(s) B_{1}^{\alpha}\left(\theta_{1}(s)\right)+q_{2}(s) B_{1}^{\alpha}\left(\theta_{2}(s)\right)\right) v^{\alpha}\left(\theta_{2}(s)\right) \\
& =-G(s) v^{\alpha}\left(\theta_{2}(s)\right)
\end{aligned}
$$

Since $\left(r(s)\left(v^{\prime}(s)\right)^{\alpha}\right)^{\prime} \leq 0$, we have

$$
r(s)\left(v^{\prime}(s)\right)^{\alpha} \leq r\left(s_{1}\right)\left(v^{\prime}\left(s_{1}\right)\right)^{\alpha}:=-L<0,
$$

for all $s \geq s_{1}$, and from (5) and (8), we have

$$
v^{\alpha}(s) \geq L \kappa^{\alpha}(s, \infty) \text { for all } s \geq s_{1} .
$$

Combining (7) with (9) yields

$$
\left(r(s)\left(v^{\prime}(s)\right)^{\alpha}\right)^{\prime} \leq-G(s) L \kappa^{\alpha}\left(\theta_{2}(s), \infty\right),
$$

for all $s \geq s_{1}$. Integrating (10) from $s_{1}$ to $s$, we obtain

$$
\begin{aligned}
r(s)\left(v^{\prime}(s)\right)^{\alpha} & \leq r\left(s_{1}\right)\left(v^{\prime}\left(s_{1}\right)\right)^{\alpha}-L \int_{s_{1}}^{s} G(\xi) \kappa^{\alpha}\left(\theta_{2}(\xi), \infty\right) \mathrm{d} \xi \\
& \leq-L \int_{s_{1}}^{s} G(\xi) \kappa^{\alpha}\left(\theta_{2}(\xi), \infty\right) \mathrm{d} \xi .
\end{aligned}
$$

Integrating the last inequality from $s_{1}$ to $s$, we get

$$
v(s) \leq v\left(s_{1}\right)-L^{1 / \alpha} \int_{s_{1}}^{s} \frac{1}{r^{1 / \alpha}(u)}\left(\int_{s_{1}}^{u} G(\xi) \kappa^{\alpha}\left(\theta_{2}(\xi), \infty\right) \mathrm{d} \xi\right)^{1 / \alpha} \mathrm{d} u .
$$

Passing to the limit as $s \rightarrow \infty$, we arrive at a contradiction with (6). Now, assume that $v^{\prime}(s)>0$ on $\left[s_{1}, \infty\right)$. From Lemma 2, we arrive at

$$
v\left(\varrho_{2}(s)\right) \leq \frac{\kappa\left(s_{1}, \varrho_{2}(s)\right)}{\kappa\left(s_{1}, s\right)} v(s)
$$

From the definition of $v$, we obtain

$$
\begin{aligned}
x(s) & =v(s)-p_{1}(s) x\left(\varrho_{1}(s)\right)-p_{2}(s) x\left(\varrho_{2}(s)\right) \\
& \geq v(s)-p_{1}(s) v\left(\varrho_{1}(s)\right)-p_{2}(s) v\left(\varrho_{2}(s)\right) .
\end{aligned}
$$

Using that (11) and $v\left(\varrho_{1}(s)\right) \leq v(s)$, where $\varrho_{1}(s)<s$ in (12), we obtain

$$
\begin{aligned}
x(s) & \geq v(s)\left(1-p_{1}(s)-p_{2}(s) \frac{\kappa\left(s_{1}, \varrho_{2}(s)\right)}{\kappa\left(s_{1}, s\right)}\right) \\
& \geq B_{2}(s) v(s) .
\end{aligned}
$$

Hence, (1) becomes

$$
\left(r(s)\left(v^{\prime}(s)\right)^{\alpha}\right)^{\prime} \leq-q_{1}(s) B_{2}^{\alpha}\left(\theta_{1}(s)\right) v^{\alpha}\left(\theta_{1}(s)\right)-q_{2}(s) B_{2}^{\alpha}\left(\theta_{2}(s)\right) v^{\alpha}\left(\theta_{2}(s)\right),
$$


and since $\theta_{1}(s) \leq \theta_{2}(s)$, we have

$$
\begin{aligned}
\left(r(s)\left(v^{\prime}(s)\right)^{\alpha}\right)^{\prime} & \leq-q_{1}(s) B_{2}^{\alpha}\left(\theta_{1}(s)\right) v^{\alpha}\left(\theta_{1}(s)\right)-q_{2}(s) B_{2}^{\alpha}\left(\theta_{2}(s)\right) v^{\alpha}\left(\theta_{1}(s)\right) \\
& \leq-\left(q_{1}(s) B_{2}^{\alpha}\left(\theta_{1}(s)\right)+q_{2}(s) B_{2}^{\alpha}\left(\theta_{2}(s)\right)\right) v^{\alpha}\left(\theta_{1}(s)\right) \\
& =-H(s) v^{\alpha}\left(\theta_{1}(s)\right) .
\end{aligned}
$$

On the other hand, it follows from (6) and (C2) that $\int_{s_{1}}^{s} G(\xi) \kappa^{\alpha}\left(\theta_{2}(\xi), \infty\right) \mathrm{d} \xi$ must be unbounded. Further, since $\kappa^{\prime}(s, \infty)<0$, it is easy to see that

$$
\int_{s_{1}}^{s} G(\xi) \mathrm{d} \xi \rightarrow \infty \text { as } s \rightarrow \infty
$$

Integrating (14) from $s_{2}$ to $s$, we get

$$
\begin{aligned}
r(s)\left(v^{\prime}(s)\right)^{\alpha} & \leq r\left(s_{2}\right)\left(v^{\prime}\left(s_{2}\right)\right)^{\alpha}-\int_{s_{2}}^{s} H(\xi) v^{\alpha}\left(\theta_{1}(\xi)\right) \mathrm{d} \xi \\
& \leq r\left(s_{2}\right)\left(v^{\prime}\left(s_{2}\right)\right)^{\alpha}-v^{\alpha}\left(\theta_{1}\left(s_{2}\right)\right) \int_{s_{2}}^{s} H(\xi) \mathrm{d} \xi .
\end{aligned}
$$

Since $H(s)>G(s)$, we get

$$
r(s)\left(v^{\prime}(s)\right)^{\alpha} \leq r\left(s_{2}\right)\left(v^{\prime}\left(s_{2}\right)\right)^{\alpha}-v^{\alpha}\left(\theta_{1}\left(s_{2}\right)\right) \int_{s_{2}}^{s} G(\xi) \mathrm{d} \xi,
$$

which, with (15), contradicts the fact that $v^{\prime}(s)>0$. The proof is complete.

Theorem 2. Assume that $H(s) \geq G(s)>0$. If

$$
\limsup _{s \rightarrow \infty} \kappa^{\alpha}\left(\theta_{2}(s), \infty\right) \int_{s_{1}}^{s} G(\xi) \mathrm{d} \xi>1,
$$

then all solutions of (1) are oscillatory.

Proof. Assume the contrary: that (1) has a non-oscillatory solution $x$ on $\left[s_{0}, \infty\right)$. Without loss of generality (since the substitution $y=-x$ transforms (1) into an equation of the same form), we suppose that $x$ is an eventually positive solution. Then, there exists $s_{1} \geq s_{0}$ such that $x\left(\varrho_{1}(s)\right)>0, x\left(\varrho_{2}(s)\right)>0, x\left(\theta_{1}(s)\right)>0$, and $x\left(\theta_{2}(s)\right)>0$ for all $s \geq s_{1}$. As in the proof of Theorem $1, v^{\prime}>0$ or $v^{\prime}<0$ eventually.

Assume that $v^{\prime}<0$ on $\left[s_{1}, \infty\right)$. Integrating (7) from $s_{1}$ to $s$, we get

$$
\begin{aligned}
r(s)\left(v^{\prime}(s)\right)^{\alpha} & \leq r\left(s_{1}\right)\left(v^{\prime}\left(s_{1}\right)\right)^{\alpha}-\int_{s_{1}}^{s} G(\xi) v^{\alpha}\left(\theta_{2}(\xi)\right) \mathrm{d} \xi \\
& \leq-v^{\alpha}\left(\theta_{2}(s)\right) \int_{s_{1}}^{s} G(\xi) \mathrm{d} \xi .
\end{aligned}
$$

Since $\theta_{2}(s) \geq s$, then from (3), we have

$$
v\left(\theta_{2}(s)\right) \geq \frac{\kappa\left(\theta_{2}(s), \infty\right)}{\kappa(s, \infty)} v(s)
$$

and using (19) and (5) in (18), we obtain

$$
r(s)\left(v^{\prime}(s)\right)^{\alpha} \leq r(s)\left(v^{\prime}(s)\right)^{\alpha} \kappa^{\alpha}\left(\theta_{2}(s), \infty\right) \int_{s_{1}}^{s} G(\xi) \mathrm{d} \xi .
$$

Dividing both sides of inequality (20) by $r(s)\left(v^{\prime}(s)\right)^{\alpha}$ and taking the limsup, we get 


$$
\limsup _{s \rightarrow \infty} \kappa^{\alpha}\left(\theta_{2}(s), \infty\right) \int_{s_{1}}^{s} G(\xi) d \xi \leq 1,
$$

we obtain a contradiction with the condition (17).

Now, assume that $v^{\prime}>0$ on $\left[s_{1}, \infty\right)$. From (17) and the fact that $\kappa\left(\theta_{2}(s), \infty\right)<\infty$, we have that (15) holds. Then, this part of the proof is similar to that of Theorem 1 . Therefore, the proof is complete.

Theorem 3. Assume that $H(s) \geq G(s)>0$ and (15) hold. Further, if the differential equation

$$
v^{\prime}(s)+\frac{1}{r^{1 / \alpha}(s)} \frac{\kappa\left(\theta_{2}(s), \infty\right)}{\kappa\left(\theta_{1}(s), \infty\right)}\left(\int_{s_{1}}^{s} G(\xi) \mathrm{d} \xi\right)^{1 / \alpha} v\left(\theta_{1}(s)\right)=0
$$

is oscillatory, then all solutions of (1) are oscillatory.

Proof. Assume the contrary: that (1) has a non-oscillatory solution $x$ on $\left[s_{0}, \infty\right)$. Without loss of generality (since the substitution $y=-x$ transforms (1) into an equation of the same form), we suppose that $x$ is an eventually positive solution. Then, there exists $s_{1} \geq s_{0}$ such that $x\left(\varrho_{1}(s)\right)>0, x\left(\varrho_{2}(s)\right)>0, x\left(\theta_{1}(s)\right)>0$, and $x\left(\theta_{2}(s)\right)>0$ for all $s \geq s_{1}$. As in the proof of Theorem $1, v^{\prime}>0$ or $v^{\prime}<0$ eventually.

Assume that $v^{\prime}<0$ on $\left[s_{1}, \infty\right)$. Since $\theta_{2}(s) \geq \theta_{1}(s)$, we get, from (3), that

$$
v\left(\theta_{2}(s)\right) \geq \frac{\kappa\left(\theta_{2}(s), \infty\right)}{\kappa\left(\theta_{1}(s), \infty\right)} v\left(\theta_{1}(s)\right)
$$

which, with (18), gives

$$
r(s)\left(v^{\prime}(s)\right)^{\alpha} \leq \frac{\kappa^{\alpha}\left(\theta_{2}(s), \infty\right)}{\kappa^{\alpha}\left(\theta_{1}(s), \infty\right)} v^{\alpha}\left(\theta_{1}(s)\right) \int_{s_{1}}^{s} G(\xi) \mathrm{d} \xi .
$$

Now, we see that $v>0$ is a solution of the inequality

$$
v^{\prime}(s)+\frac{1}{r^{1 / \alpha}(s)} \frac{\kappa\left(\theta_{2}(s), \infty\right)}{\kappa\left(\theta_{1}(s), \infty\right)}\left(\int_{s_{1}}^{s} G(\xi) \mathrm{d} \xi\right)^{1 / \alpha} v\left(\theta_{1}(s)\right) \leq 0
$$

Using [34], we find that (21) also has a positive solution-a contradiction. By proceeding as in the proof of Theorem 1, the proof of this theory is completed.

Corollary 1. Assume that $H(s) \geq G(s)>0$ and (15) hold. If

$$
\liminf _{s \rightarrow \infty} \int_{\theta_{1}(s)}^{s} \frac{1}{r^{1 / \alpha}(u)} \frac{\kappa\left(\theta_{2}(u), \infty\right)}{\kappa\left(\theta_{1}(u), \infty\right)}\left(\int_{s_{1}}^{u} G(\xi) \mathrm{d} \xi\right)^{1 / \alpha} \mathrm{d} u>\frac{1}{\mathrm{e}^{\prime}}
$$

then all solutions of (1) are oscillatory.

Proof. Using ([35], Theorem 2), we have that (22) implies the oscillation of (21). From Theorem 3, we have that (1) is oscillatory.

Theorem 4. Assume that $H(s)>0, G(s)>0$. If there exist functions $\psi, \delta \in C^{1}\left(\left[s_{0}, \infty\right),(0, \infty)\right)$, and $s_{1} \in\left[s_{0}, \infty\right)$ such that

$$
\limsup _{s \rightarrow \infty}\left\{\frac{\kappa^{\alpha}(s, \infty)}{\delta(s)} \int_{s_{1}}^{s}\left(\delta(\xi) G(\xi) \frac{\kappa^{\alpha}\left(\theta_{2}(\xi), \infty\right)}{\kappa^{\alpha}(\xi, \infty)}-\frac{r(\xi)\left(\delta^{\prime}(\xi)\right)^{\alpha+1}}{(\alpha+1)^{\alpha+1}(\delta(\xi))^{\alpha}}\right) \mathrm{d} \xi\right\}>1
$$


and

$$
\limsup _{s \rightarrow \infty} \int_{s_{1}}^{s}\left(\psi(\xi) H(\xi)-\frac{1}{(\alpha+1)^{\alpha+1}} \frac{r(\xi)\left(\psi^{\prime}(\xi)\right)^{\alpha+1}}{\psi^{\alpha}(\xi)\left(\theta_{1}^{\prime}(\xi)\right)^{\alpha}}\right) d \xi=\infty,
$$

then all solutions of (1) are oscillatory.

Proof. Assume the contrary: that (1) has a non-oscillatory solution $x$ on $\left[s_{0}, \infty\right)$. Without loss of generality (since the substitution $y=-x$ transforms (1) into an equation of the same form), we suppose that $x$ is an eventually positive solution. Then, there exists $s_{1} \geq s_{0}$ such that $x\left(\varrho_{1}(s)\right)>0, x\left(\varrho_{2}(s)\right)>0, x\left(\theta_{1}(s)\right)>0$, and $x\left(\theta_{2}(s)\right)>0$ for all $s \geq s_{1}$. From Theorem $1, v^{\prime}>0$ or $v^{\prime}<0$ eventually.

Assume that $v^{\prime}<0$ on $\left[s_{1}, \infty\right)$. As in the proof of Theorem 1 , we arrive at (7). Now, we define the function

$$
\omega(s)=\delta(s)\left(\frac{r(s)\left(v^{\prime}(s)\right)^{\alpha}}{v^{\alpha}(s)}+\frac{1}{\kappa^{\alpha}(s, \infty)}\right) \text { on }\left[s_{1}, \infty\right) .
$$

From (5), we get that $\omega \geq 0$ on $\left[s_{1}, \infty\right)$. Differentiating (25), we obtain

$$
\begin{aligned}
\omega^{\prime}(s)= & \frac{\delta^{\prime}(s)}{\delta(s)} \omega(s)+\delta(s) \frac{\left(r(s)\left(v^{\prime}(s)\right)^{\alpha}\right)^{\prime}}{v^{\alpha}(s)}-\alpha \delta(s) r(s)\left(\frac{v^{\prime}(s)}{v(s)}\right)^{\alpha+1} \\
& +\frac{\alpha \delta(s)}{r^{1 / \alpha}(s) \kappa^{\alpha+1}(s, \infty)} \\
\leq & \frac{\delta^{\prime}(s)}{\delta(s)} \omega(s)+\delta(s) \frac{\left(r(s)\left(v^{\prime}(s)\right)^{\alpha}\right)^{\prime}}{v^{\alpha}(s)}-\frac{\alpha}{(\delta(s) r(s))^{1 / \alpha}}\left(\omega(s)-\frac{\delta(s)}{\kappa^{\alpha}(s, \infty)}\right)^{(\alpha+1) / \alpha} \\
& +\frac{\alpha \delta(s)}{r^{1 / \alpha}(s) \kappa^{\alpha+1}(s, \infty)} .
\end{aligned}
$$

Combining (7) and (26), we have

$$
\begin{aligned}
\omega^{\prime}(s) \leq & -\frac{\alpha}{(\delta(s) r(s))^{1 / \alpha}}\left(\omega(s)-\frac{\delta(s)}{\kappa^{\alpha}(s, \infty)}\right)^{(\alpha+1) / \alpha}-\delta(s) G(s) \frac{v^{\alpha}\left(\theta_{2}(s)\right)}{v^{\alpha}(s)} \\
& +\frac{\alpha \delta(s)}{r^{1 / \alpha}(s) \kappa^{\alpha+1}(s, \infty)}+\frac{\delta^{\prime}(s)}{\delta(s)} \omega(s) .
\end{aligned}
$$

Using Lemma 1 with $A:=\delta^{\prime}(s) / \delta(s), B:=\alpha(\delta(s) r(s))^{-1 / \alpha}, C:=\delta(s) / \kappa^{\alpha}(s, \infty)$ and $\xi:=\omega$, we get

$$
\begin{aligned}
\frac{\delta^{\prime}(s)}{\delta(s)} \omega(s)-\frac{\alpha}{(\delta(s) r(s))^{1 / \alpha}}\left(\omega(s)-\frac{\delta(s)}{\kappa^{\alpha}(s, \infty)}\right)^{(\alpha+1) / \alpha} \leq & \frac{r(s)}{(\alpha+1)^{\alpha+1}} \frac{\left(\delta^{\prime}(s)\right)^{\alpha+1}}{(\delta(s))^{\alpha}} \\
& +\frac{\delta^{\prime}(s)}{\mathcal{\kappa}^{\alpha}(s, \infty)}
\end{aligned}
$$

and since $s \leq \theta_{2}(s)$, we arrive at

$$
v\left(\theta_{2}(s)\right) \geq \frac{\kappa\left(\theta_{2}(s), \infty\right)}{\kappa(s, \infty)} v(s)
$$

which, in view of (27), (28), and (29), gives 


$$
\begin{aligned}
\omega^{\prime}(s) \leq & \frac{\delta^{\prime}(s)}{\mathcal{\kappa}^{\alpha}(s, \infty)}+\frac{1}{(\alpha+1)^{\alpha+1}} r(s) \frac{\left(\delta^{\prime}(s)\right)^{\alpha+1}}{(\delta(s))^{\alpha}}-\delta(s) G(s) \frac{v^{\alpha}\left(\theta_{2}(s)\right)}{v^{\alpha}(s)} \\
& +\frac{\alpha \delta(s)}{r^{1 / \alpha}(s) \kappa^{\alpha+1}(s, \infty)} \\
\leq & -\delta(s) G(s) \frac{\kappa^{\alpha}\left(\theta_{2}(s), \infty\right)}{\kappa^{\alpha}(s, \infty)}+\left(\frac{\delta(s)}{\kappa^{\alpha}(s, \infty)}\right)^{\prime}+\frac{r(s)\left(\delta^{\prime}(s)\right)^{\alpha+1}}{(\alpha+1)^{\alpha+1}(\delta(s))^{\alpha}} .
\end{aligned}
$$

Integrating (30) from $s_{2}$ to $s$, we arrive at

$$
\begin{aligned}
\int_{s_{2}}^{s}\left(\delta(\xi) G(\xi) \frac{\kappa^{\alpha}\left(\theta_{2}(\xi), \infty\right)}{\kappa^{\alpha}(\xi, \infty)}-\frac{r(\xi)\left(\delta^{\prime}(\xi)\right)^{\alpha+1}}{(\alpha+1)^{\alpha+1}(\delta(\xi))^{\alpha}}\right) \mathrm{d} \xi & \leq\left.\left(\frac{\delta(s)}{\kappa^{\alpha}(s, \infty)}-\omega(s)\right)\right|_{s_{2}} ^{s} \\
& \leq-\left.\left(\delta(s) \frac{r(s)\left(v^{\prime}(s)\right)^{\alpha}}{v^{\alpha}(s)}\right)\right|_{s_{2}} ^{s}(31)
\end{aligned}
$$

From (5), we have

$$
-\frac{r^{1 / \alpha}(s) v^{\prime}(s)}{v(s)} \leq \frac{1}{\kappa(s, \infty)}
$$

which, in view of (31), implies

$$
\frac{\kappa^{\alpha}(s, \infty)}{\delta(s)} \int_{s_{2}}^{s}\left(\delta(\xi) G(\xi) \frac{\kappa^{\alpha}\left(\theta_{2}(\xi), \infty\right)}{\kappa^{\alpha}(\xi, \infty)}-\frac{r(\xi)\left(\delta^{\prime}(\xi)\right)^{\alpha+1}}{(\alpha+1)^{\alpha+1}(\delta(\xi))^{\alpha}}\right) \mathrm{d} \xi \leq 1 .
$$

Thus, we get a contradiction with (23).

Now, assume that $v^{\prime}(s)>0$ on $\left[s_{1}, \infty\right)$. Let us define the Riccati function

$$
\varphi(s)=\psi(s) \frac{r(s)\left(v^{\prime}(s)\right)^{\alpha}}{v^{\alpha}\left(\theta_{1}(s)\right)}, \text { on }\left[s_{1}, \infty\right)
$$

We find that $\varphi \geq 0$ on $\left[s_{1}, \infty\right)$. Differentiating (32), we get

$$
\varphi^{\prime}(s)=\frac{\psi^{\prime}(s)}{\psi(s)} \varphi(s)+\psi(s) \frac{\left(r(s)\left(v^{\prime}(s)\right)^{\alpha}\right)^{\prime}}{v^{\alpha}\left(\theta_{1}(s)\right)}-\alpha \psi(s) r(s) \frac{\left(v^{\prime}(s)\right)^{\alpha} v^{\prime}\left(\theta_{1}(s)\right) \theta_{1}^{\prime}(s)}{v^{\alpha+1}\left(\theta_{1}(s)\right)} .
$$

Combining (14) and (33), we have

$$
\varphi^{\prime}(s) \leq \frac{\psi^{\prime}(s)}{\psi(s)} \varphi(s)-\psi(s) H(s)-\alpha \psi(s) r(s) \frac{\left(v^{\prime}(s)\right)^{\alpha} v^{\prime}\left(\theta_{1}(s)\right) \theta_{1}^{\prime}(s)}{v^{\alpha+1}\left(\theta_{1}(s)\right)} .
$$

Since $\left(r(s)\left(v^{\prime}(s)\right)^{\alpha}\right)^{\prime}<0$ and $\theta_{1}(s) \leq s$, we arrive at

$$
\varphi^{\prime}(s) \leq \frac{\psi^{\prime}(s)}{\psi(s)} \varphi(s)-\psi(s) H(s)-\alpha \psi(s) r(s) \theta_{1}^{\prime}(s) \frac{\left(v^{\prime}(s)\right)^{\alpha+1}}{v^{\alpha+1}\left(\theta_{1}(s)\right)},
$$

and from (32), we have

$$
\varphi^{\prime}(s) \leq \frac{\psi^{\prime}(s)}{\psi(s)} \varphi(s)-\psi(s) H(s)-\frac{\alpha \theta_{1}^{\prime}(s)}{\psi^{1 / \alpha}(s) r^{1 / \alpha}(s)} \phi^{(\alpha+1) / \alpha}(s) .
$$

Using the inequality

$$
K v-s v^{(\alpha+1) / \alpha} \leq \frac{\alpha^{\alpha}}{(\alpha+1)^{\alpha+1}} \frac{K^{\alpha+1}}{s^{\alpha}}, s>0,
$$


with $K=\psi^{\prime}(s) / \psi(s), s=\alpha \theta_{1}^{\prime}(s) / \psi^{1 / \alpha}(s) r^{1 / \alpha}(s)$, and $v=\varphi$, we have

$$
\varphi^{\prime}(s) \leq-\psi(s) H(s)+\frac{1}{(\alpha+1)^{\alpha+1}} \frac{r(s)\left(\psi^{\prime}(s)\right)^{\alpha+1}}{\psi^{\alpha}(s)\left(\theta_{1}^{\prime}(s)\right)^{\alpha}} .
$$

Integrating (35) from $s_{2}$ to $s$, we arrive at

$$
\int_{s_{2}}^{s}\left(\psi(\xi) H(\xi)-\frac{1}{(\alpha+1)^{\alpha+1}} \frac{r(\xi)\left(\psi^{\prime}(\xi)\right)^{\alpha+1}}{\psi^{\alpha}(\xi)\left(\theta_{1}^{\prime}(\xi)\right)^{\alpha}}\right) \mathrm{d} \xi \leq \varphi\left(s_{2}\right) .
$$

Taking the limsup on both sides of this inequality, we have a contradiction with (24). The proof of the theorem is complete.

Theorem 5. Assume that $H(s)>0$ and $G(s)>0$. If there exist the functions $\delta \in C^{1}\left(\left[s_{0}, \infty\right),(0, \infty)\right)$ and $s_{1} \in\left[s_{0}, \infty\right)$ such that (23) and

$$
\liminf _{s \rightarrow \infty} \frac{\alpha}{\Psi(s)} \int_{s}^{\infty} \frac{\theta_{1}^{\prime}(\xi)}{r^{1 / \alpha}(\xi)} \Psi^{(\alpha+1) / \alpha}(\xi) \mathrm{d} \xi>\frac{\alpha}{(\alpha+1)^{(\alpha+1) / \alpha}}
$$

hold, where

$$
\Psi(s)=\int_{s}^{\infty} H(\xi) \mathrm{d} \xi,
$$

then all solutions of (1) are oscillatory.

Proof. Assume the contrary: that (1) has a non-oscillatory solution $x$ on $\left[s_{0}, \infty\right)$. Without loss of generality (since the substitution $y=-x$ transforms (1) into an equation of the same form), we suppose that $x$ is an eventually positive solution. Then, there exists $s_{1} \geq s_{0}$ such that $x\left(\varrho_{1}(s)\right)>0, x\left(\varrho_{2}(s)\right)>0, x\left(\theta_{1}(s)\right)>0$, and $x\left(\theta_{2}(s)\right)>0$ for all $s \geq s_{1}$. Theorem 1 yields that $v^{\prime}$ eventually has one sign.

Assume that $v^{\prime}(s)<0$ on $\left[s_{1}, \infty\right)$. The proof is similar to that of Theorem 4 . Now, assume that $v^{\prime}(s)>0$ on $\left[s_{1}, \infty\right)$. Let us define the Riccati function

$$
\varphi(s)=\frac{r(s)\left(v^{\prime}(s)\right)^{\alpha}}{v^{\alpha}\left(\theta_{1}(s)\right)} .
$$

We see that $\varphi \geq 0$ on $\left[s_{1}, \infty\right)$. Differentiating (37), we arrive at

$$
\varphi^{\prime}(s)=\frac{\left(r(s)\left(v^{\prime}(s)\right)^{\alpha}\right)^{\prime}}{v^{\alpha}\left(\theta_{1}(s)\right)}-\alpha r(s) \frac{\left(v^{\prime}(s)\right)^{\alpha} v^{\prime}\left(\theta_{1}(s)\right) \theta_{1}^{\prime}(s)}{v^{\alpha+1}\left(\theta_{1}(s)\right)} .
$$

Combining (14) and (38), we have

$$
\varphi^{\prime}(s) \leq-H(s)-\alpha r(s) \frac{\left(v^{\prime}(s)\right)^{\alpha} v^{\prime}\left(\theta_{1}(s)\right) \theta_{1}^{\prime}(s)}{v^{\alpha+1}\left(\theta_{1}(s)\right)} .
$$

Since $\left(r(s)\left(v^{\prime}(s)\right)^{\alpha}\right)^{\prime}<0$ and $\theta_{1}(s) \leq s$, we arrive at

$$
\varphi^{\prime}(s) \leq-H(s)-\alpha r(s) \theta_{1}^{\prime}(s) \frac{\left(v^{\prime}(s)\right)^{\alpha+1}}{v^{\alpha+1}\left(\theta_{1}(s)\right)},
$$

which, with (37), gives

$$
\varphi^{\prime}(s) \leq-H(s)-\frac{\alpha \theta_{1}^{\prime}(s)}{r^{1 / \alpha}(s)} \varphi^{(\alpha+1) / \alpha}(s)
$$

Integrating (39) from $s$ to $\infty$, and using the fact that $\varphi(s)>0$ and $\varphi^{\prime}(s)<0$, we get 


$$
-\varphi(s) \leq-\int_{s}^{\infty} H(\xi) \mathrm{d} \xi-\int_{s}^{\infty} \frac{\alpha \theta_{1}^{\prime}(\xi)}{r^{1 / \alpha}(\xi)} \varphi^{(\alpha+1) / \alpha}(\xi) \mathrm{d} \xi .
$$

Hence, we have

$$
\frac{\varphi(s)}{\Psi(s)} \geq 1+\frac{1}{\Psi(s)} \int_{s}^{\infty} \frac{\alpha \theta_{1}^{\prime}(\xi)}{r^{1 / \alpha}(\xi)} \Psi^{(\alpha+1) / \alpha}(\xi)\left(\frac{\varphi(\xi)}{\Psi(\xi)}\right)^{(\alpha+1) / \alpha} \mathrm{d} \xi .
$$
(36) that

Let $\vartheta=\inf _{s \geq s} \varphi(s) / \Psi(s)$; then, obviously, $\vartheta \geq 1$. Hence, it follows from (40) and

$$
\vartheta \geq 1+\alpha\left(\frac{\vartheta}{\alpha+1}\right)^{(\alpha+1) / \alpha}
$$

or

$$
\frac{\vartheta}{\alpha+1} \geq \frac{1}{\alpha+1}+\frac{\alpha}{\alpha+1}\left(\frac{\vartheta}{\alpha+1}\right)^{(\alpha+1) / \alpha}
$$

which contradicts the admissible value of $\vartheta$ and $\alpha$. Therefore, the proof is complete.

Corollary 2. Assume that $H(s)>0$ and $G(s)>0$. If (36) and either

$$
\begin{gathered}
\limsup _{s \rightarrow \infty} \int_{s}^{s}\left(G(\xi) \kappa^{\alpha}\left(\theta_{2}(\xi), \infty\right)-\frac{\alpha^{\alpha+1}}{(\alpha+1)^{\alpha+1} r^{1 / \alpha}(\xi) \kappa(\xi, \infty)}\right) d \xi>1, \\
\limsup _{s \rightarrow \infty} \kappa^{\alpha-1}(s, \infty) \int_{s}^{s}\left(G(\xi) \frac{\kappa^{\alpha}\left(\theta_{2}(\xi), \infty\right)}{\kappa^{\alpha-1}(\xi, \infty)}-\frac{1}{(\alpha+1)^{\alpha+1} r^{1 / \alpha}(\xi) \kappa^{\alpha}(\xi, \infty)}\right) d \xi>1,
\end{gathered}
$$

or

$$
\limsup _{s \rightarrow \infty} \kappa^{\alpha}(s, \infty) \int_{s}^{s} G(\xi) \frac{\kappa^{\alpha}\left(\theta_{2}(\xi), \infty\right)}{\kappa^{\alpha}(\xi, \infty)} \mathrm{d} \xi>1,
$$

hold, then all solutions of (1) are oscillatory.

Proof. By choosing $\delta(s)=\kappa^{\alpha}(s, \infty), \delta(s)=\kappa(s, \infty)$, or $\delta(s)=1$, the condition (23) reduces to one of the conditions (41)-(43), respectively.

Example 1. Consider the second-order neutral differential equation

$$
\left(s^{2}\left(x(s)+p_{1}^{*} x\left(\frac{s}{\lambda}\right)+p_{2}^{*} x(\lambda s)\right)^{\prime}\right)^{\prime}+q_{1}^{*} x\left(\frac{s}{\mu}\right)+q_{2}^{*} x(\mu s)=0,
$$

where $s \geq 1, \lambda \geq 1, \mu \geq 1, p_{1}^{*}>p_{2}^{*}$, and $\lambda\left(p_{1}^{*}+p_{2}^{*}\right) \in(0,1)$. Now, we note that $r(s)=s^{2}$, $p_{1}(s)=p_{1}^{*}, p_{2}(s)=p_{2}^{*}, \varrho_{1}(s)=s / \lambda, \varrho_{2}(s)=\lambda s, q_{1}(s)=q_{1}^{*}, q_{2}(s)=q_{2}^{*}, \theta_{1}(s)=s / \mu$, and $\theta_{2}(s)=\mu s$.Thus, we have that

$$
B_{1}(s)=1-\lambda p_{1}^{*}-p_{2}^{*}, \quad B_{2}(s)=1-p_{1}^{*}-p_{2}^{*}\left(\frac{s-\frac{1}{\lambda}}{s-1}\right)
$$

and

$$
G(s)=\left(q_{1}^{*}+q_{2}^{*}\right)\left(1-\lambda p_{1}^{*}-p_{2}^{*}\right) .
$$

Set $W(s)=\left(s-\frac{1}{\lambda}\right) /(s-1)$. Since $\lim _{s \rightarrow \infty} W(s)=1$, there exists $s_{\epsilon}>s_{0}$ such that $W(s)<1+\epsilon$ for all $\epsilon>0$ and every $s \geq s_{\epsilon}$. By choosing $\epsilon=\lambda-1$, we obtain $W(s)<\lambda$ for all $s \geq s_{*}$. Thus, and taking into account the fact that $p_{1}^{*}>p_{2}^{*}$ and $\lambda p_{1}^{*}+p_{2}^{*} \in(0,1)$, we get that $B_{2} \geq B_{1}>0$. Now, from Theorem 2 , we have that equation (44) is oscillatory if

$$
q_{1}^{*}+q_{2}^{*}>\frac{\mu}{1-\lambda p_{1}^{*}-p_{2}^{*}} .
$$


On the other hand, using Corollary 1, we see that (44) is oscillatory if

$$
q_{1}^{*}+q_{2}^{*}>\frac{\mu}{\left(1-\lambda p_{1}^{*}-p_{2}^{*}\right)} \frac{\mu}{\mathrm{e} \ln \mu} .
$$

Next, since $W(s)<\lambda$ for all $s \geq s_{*}$, we find that $B_{2}(s)>1-\left(p_{1}^{*}+\lambda p_{2}^{*}\right)$, and so, $H(s)>\left(q_{1}^{*}+q_{2}^{*}\right)\left(1-\left(p_{1}^{*}+\lambda p_{2}^{*}\right)\right)$. Hence, by choosing $\psi(s)=1$, condition (24) holds, directly. Using Theorem 4, we see that (44) is oscillatory if

$$
q_{1}^{*}+q_{2}^{*}>\frac{1}{4} \frac{\mu}{\left(1-\lambda p_{1}^{*}-p_{2}^{*}\right)}
$$

Remark 1. Taking the fact that $\mu>e \ln \mu$ into account, it is easy to notice that condition (C3) supports the most efficient condition for oscillation of (44). Figures 1 and 2 display a comparison of the criteria (C1)-(C3).

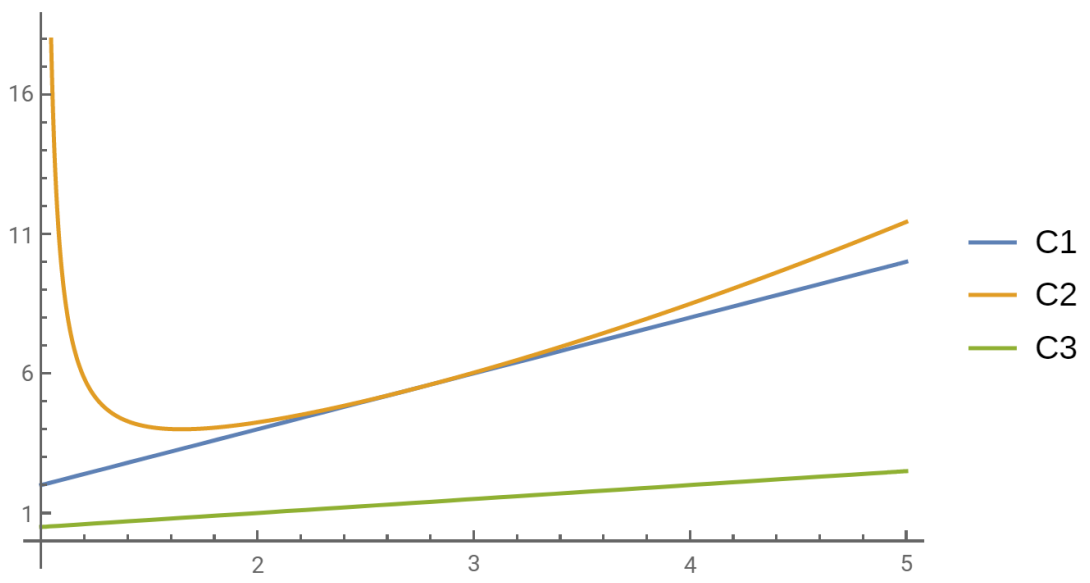

Figure 1. Comparison of the criteria (C1)-(C3) when $\lambda=2, p_{1}^{*}=0.25$, and $p_{2}^{*}=0$

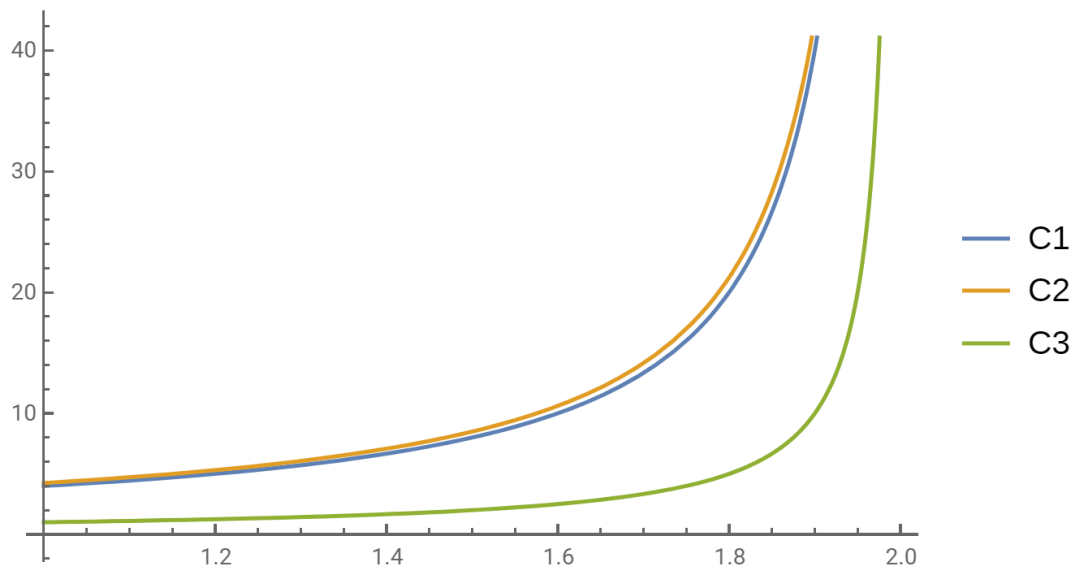

Figure 2. Comparison of the criteria (C1)-(C3) when $\mu=2, p_{1}^{*}=0.5$, and $p_{2}^{*}=0$

Remark 2. In the special case of $(44), p_{2}^{*}=q_{2}^{*}=0$, that is,

$$
\left(s^{2}\left(x(s)+p_{1}^{*} x\left(\frac{s}{\lambda}\right)\right)^{\prime}\right)^{\prime}+q_{1}^{*} x\left(\frac{s}{\mu}\right)=0 .
$$

The oscillation criterion (C3) reduces to 


$$
q_{1}^{*}>\frac{1}{4} \frac{\mu}{\left(1-\lambda p_{1}^{*}\right)},
$$

which is the exact criterion that was obtained in Example 3.1 in [7]. Moreover, if $p_{1}^{*}=0$ and $\mu=1$, then condition (45) reduces so that $q_{1}^{*}>1 / 4$, which is a sharp condition for oscillation of the second-order Euler equation.

\section{Conclusions}

Most works that studied the oscillatory behavior of mixed equations regarded the canonical case $\pi\left(l_{0}\right)=\infty$. Likewise, works that were concerned with the non-canonical case of neutral equations obtained two conditions for testing the oscillation. In this paper, we focused on studying the non-canonical case, and we created criteria with only one condition that is easy to verify. Therefore, our results are an extension, complement, and improvement to previous results in the literature. It is interesting to extend the results of this paper to higher-order equations.

Author Contributions: Formal analysis, O.M., A.M. and S.S.S.; Investigation, O.M., A.M. and S.S.S.; Methodology, O.M.; Writing-original draft, A.M. and S.S.S.; Writing-review and editing, A.M. and O.M. All authors have read and agreed to the published version of the manuscript.

Funding: There was no external funding for this article.

Acknowledgments: The authors present their sincere thanks to the editors and two anonymous referees.

Conflicts of Interest: The authors declare no conflicts of interest.

\section{References}

1. Baculikova, B.; Dzurina, J. Oscillation theorems for second-order nonlinear neutral differential equations. Comput. Math. Appl. 2011, 62, 4472-4478. [CrossRef]

2. Grace, S.R.; Dzurina, J.; Jadlovska, I.; Li, T. An improved approach for studying oscillation of second-order neutral delay differential equations. J. Inequal. Appl. 2018, 2018, 193. [CrossRef] [PubMed]

3. Moaaz, O. New criteria for oscillation of nonlinear neutral differential equations. Adv. Differ. Eqs. 2019, 2019, 484. [CrossRef]

4. Moaaz, O.; Anis, M.; Baleanu, D.; Muhib, A. More effective criteria for oscillation of second-order differential equations with neutral arguments. Mathematics 2020, 8, 986. [CrossRef]

5. Xu, R.; Meng, F. Some new oscillation criteria for second order quasi-linear neutral delay differential equations. Appl. Math. Comput. 2006, 182, 797-803. [CrossRef]

6. Bohner, M.; Grace, S.R.; Jadlovska, I. Oscillation criteria for second-order neutral delay differential equations. Electron. J. Qual. Theory Differ. Equ. 2017, 60, 1-12.

7. Agarwal, R.P.; Zhang, Ch.; Li, T. Some remarks on oscillation of second order neutral differential equations. Appl. Math. Compt. 2016, 274, 178-181. [CrossRef]

8. Han, Z.; Li, T.; Sun, S.; Sun, Y. Remarks on the paper [Appl. Math. Comput. 207 (2009)388-396]. Appl. Math. Comput. 2010, 215 3998-4007. [CrossRef]

9. Bohner, M.; Grace, S.R.; Jadlovska, I. Sharp oscillation criteria for second-order neutral delay differential equations. Math. Meth. Appl. Sci. 2020, 43, 10041-10053. [CrossRef]

10. Dzurina, J.; Grace, S.R.; Jadlovska, I. Li, T. Oscillation criteria for second-order Emden-Fowler delay differential equations with a sublinear neutral term. Math. Nachr. 2020, 293, 910-922. [CrossRef]

11. Chatzarakis, G.E.; Dzurina, J.; Jadlovská, I. New oscillation criteria for second-order half-linear advanced differential equations. Appl. Math. Comput. 2019, 347, 404-416. [CrossRef]

12. Chatzarakis, G.E.; Moaaz, O.; Li, T.; Qaraad, B. Some oscillation theorems for nonlinear second-order differential equations with an advanced argument. Adv. Differ. Eqs. 2020, 2020, 160. [CrossRef]

13. Arul, R.; Shobha, VS. Oscillation of second order nonlinear neutral differential equations with mixed neutral term. J. Appl. Math. Phys. 2015, 3, 1080-1089. [CrossRef]

14. Li, T. Comparison theorems for second-order neutral differential equations of mixed type. Electron. J. Differ. Equ. 2010, 167, 1-7.

15. Li, T.; Baculíkova, B.; Dzurina, J. Oscillation results for second-order neutral differential equations of mixed type. Tatra Mt. Math. Publ. 2011, 48, 101-116. [CrossRef] 
16. Han, Z.; Li, T.; Zhang, C. Oscillation criteria for certain second-order nonlinear neutral differential equations of mixed type. In Abstract and Applied Analysis; Hindawi: London, UK, 2011; Volume 2011, pp. 1-9.

17. Grace, S.R. Oscillations of mixed neutral functional differential equations. Appl. Math. Comput. 1995, 68, 1-13. [CrossRef]

18. Qi, Y.; Yu, J. Oscillation of second order nonlinear mixed neutral differential equations with distributed deviating arguments. Bull Malays Math. Sci. Soc. 2015, 38, 543-560. [CrossRef]

19. Thandapani, E.; Padmavathi, S.; Pinelas, P. Oscillation criteria for even-order nonlinear neutral differential equations of mixed type. Bull Math Anal. Appl. 2014, 6, 9-22.

20. Yan, J. Oscillations of higher order neutral differential equations of mixed type. Israel J. Math. 2000, 115, 125-136. [CrossRef]

21. Zhang, C.; Baculíkova, B.; Dzurina, J.; Tongxing, L. Oscillation results for second-order mixed neutral differential equations with distributed deviating arguments. Math. Slovaca 2016, 66, 615-626. [CrossRef]

22. Dzurina, J.; Busa, J.; Airyan, E.A. Oscillation criteria for second-order differential equations of neutral type with mixed arguments. Differ. Equ. 2002, 38, 137-140. [CrossRef]

23. Thandapani, E.; Selvarangam, S.; Vijaya, M.; Rama, R. Oscillation Results for Second Order Nonlinear Differential Equation with Delay and Advanced Arguments. Kyungpook Math. J. 2016, 56, 137-146. [CrossRef]

24. Tunc, E.; Ozdemir, O. On the oscillation of second-order half-linear functional differential equations with mixed neutral term. J. Taibah Univ. Sci. 2019, 13, 481-489. [CrossRef]

25. Bazighifan, O.; Moaaz, O.; El-Nabulsi, R.A.; Muhib, A. Some new oscillation results for fourth-order neutral differential equations with delay argument. Symmetry 2020, 12, 1248. [CrossRef]

26. Moaaz, O.; Baleanu, D.; Muhib, A. New aspects for non-existence of kneser solutions of neutral differential equations with odd-order. Mathematics 2020, 8, 494. [CrossRef]

27. Moaaz, O.; Park, Ch.; Muhib, A.; Bazighifan, O. Oscillation criteria for a class of even-order neutral delay differential equations. J. Appl. Math. Comput. 2020, 63, 607-617. [CrossRef]

28. Moaaz, O.; Furuichi, S.; Muhib, A. New comparison theorems for the nth order neutral differential equations with delay inequalities. Mathematics 2020, 8, 454. [CrossRef]

29. Wang, P.; Teo, K. L; Liu, Y. Oscillation properties for even order neutral equations with distributed deviating arguments. J. Comput. Appl. Math. 2005, 182, 290-303. [CrossRef]

30. Wang, P.; Shi, W. Oscillatory theorems of a class of even-order neutral equations. Appl. Math. Lett. 2003, 16, 1011-1018. [CrossRef]

31. Zhang, M.; Song, G. Oscillation theorems for even order neutral equations with continuous distributed deviating arguments. Int. J. Inf. Syst. Sci. 2011, 7, 124-130.

32. Zhang, Q.; Yan, J.; Gao, L. Oscillation behavior of even-order nonlinear neutral differential equations with variable coefficients. Comput. Math. Appl. 2010, 59, 426-430. [CrossRef]

33. Zhang, S.; Meng, F. Oscillation criteria for even order neutral equations with distributed deviating argument. Int. J. Differ. Equ. 2010, 2010, 308357. [CrossRef]

34. Philos, C. On the existence of nonoscillatory solutions tending to zero at $\infty$ for differential equations with positive delay. Arch. Math. (Basel) 1981, 36, 168-178. [CrossRef]

35. Kitamura, Y.; Kusano, T. Oscillation of first-order nonlinear differential equations wit deviating arguments. Proc. Amer. Math. Soc. 1980, 78, 64-68. [CrossRef] 